\title{
A Molecular Algorithm for Path Self-Assembly in 3 Dimensions
}

\author{
Rebecca Schulman \\ Department of Physics \\ University of California, Berkeley \\ Berkeley, CA, USA \\ Email: rschulman@berkeley.edu
}

\author{
Bernard Yurke \\ Materials Science and Engineering Department \& \\ Electrical Engineering Department \\ Boise State University \\ Boise, ID, USA \\ Email: bernardyurke@boisestate.edu
}

\begin{abstract}
A molecular algorithm is a set of molecular interactions that carry out a particular task. We describe a molecular algorithm for self-assembling a path between two stationary points when the locations of these points are not known in advance. While efficient path finding algorithms for electronic robots exist, molecules lack the centralized memory or computing power to implement them. The algorithm takes advantage of the inherent physics at the molecular scale, and unlike other biomimetic algorithms for path finding, is designed to work in an unstructured environment and does not require complex molecular components. Designed molecules self-assemble a DNA nanotube starting from a "seed" molecule attached to the start point. During growth, the DNA nanotube's end diffuses through space, and this diffusion is harnessed as a search process: when the DNA nanotube's end contacts the destination, it attaches to it and stops growing, forming a stable path.

We use simulations and analysis to predict that paths of up 10 microns are formed with more than $99 \%$ probability if the destination is larger than $\mathbf{5 0 0}$ nanometers in diameter, making it practical. However, the probability of the DNA nanotube missing the destination is highly dependent on destination distance and size. It increases as $\exp \left[-1 / \Omega\left(r^{3}\right)\right]$, where $r$ is the Euclidean distance from the start point to the destination, and scales approximately as $\exp [-\Omega(d)]$, where $d$ is the diameter of the destination. A path finding algorithm that could work over longer distances or with small destinations will likely require new molecular components; we describe how new components could be used to solve the molecular path finding problem using a divide and conquer approach.
\end{abstract}

\section{INTRODUCTION}

Bottom-up construction is an increasingly promising way to inexpensively create materials with designed nanoscale features $[46,21]$. In a bottom-up construction process, designed molecules autonomously assemble the desired device. Molecules, unlike electronic devices, have almost no capacity for computation or information storage. Instead, a series of stochastic molecular attachments and detachments must collectively converge on the desired structure. These collective actions, i.e. molecular algorithms, are surprisingly powerful: molecules can perform universal construction [43] and universal computation [32,11]. Molecular algorithms are also capable of error-correction and robust behavior [10]. While in practice most are still fragile and scaling is difficult $[40,2,13]$, simple algorithms have been demonstrated experimentally. For example, designed molecules can self-assemble a Sierpinski gasket [36], and 2- and 3-dimensional structures with arbitrary features [34, 13], and can perform tasks such as walking [5, $30,23]$ and cargo transfer [16].

One way to make a molecular algorithm robust is to make it adaptive. Scaling is more feasible if individual construction algorithms (i.e. modules) can respond to errors made in the execution of a previous construction process. Environmentally adaptive molecular algorithms would be useful in unstructured environments such as cell cultures where the geometry may be uncertain and algorithms that can deduce and respond to these geometries would be required. Biological algorithms for assembly are environmentally adaptive-cells use chemical and mechanical signals to determine when and where to grow and move. However, devising ways for synthetic molecules to collectively sense the local environment and act on the results of the information received can be difficult.

In this paper we describe a molecular algorithm for path finding and characterize its performance. In the path finding problem, the inputs are fixed start and destination points of unknown location, and the desired output is a physical link between them. An algorithm to solve the path finding problem is inherently environmentally adaptive: the location of the path that is formed depends on the locations of the start and destination in the environment. Path finding is also a basic primitive for bottom-up construction and could be used to assemble wires such that they connect to both contacts when the location of the contacts is uncertain, or to immobilize an object by creating a physical tether between the object and a surface.

Biology provides an example bottom-up path finding algorithm, the separation of chromosomes during somatic animal cell division [18] that occurs through the assembly of the mitotic spindle. After a cell's chromosomes are duplicated, they remain grouped at the spindle equator. They then must be separated such that the mother and daughter cells each receive one set. A pair of kinetochores form on each pair of chromosomes and centromeres arise on either side of the chromosomes. Microtubules, nanotubes assembled from protein monomers, grow from the centromeres. If a growing microtubule encounters a kinetochore, it stays put, forming a permanent link. The kinetochores are oriented on pairs of chromosomes such that the two attaching microtubules come from different centromeres. The centromeres pull on the 


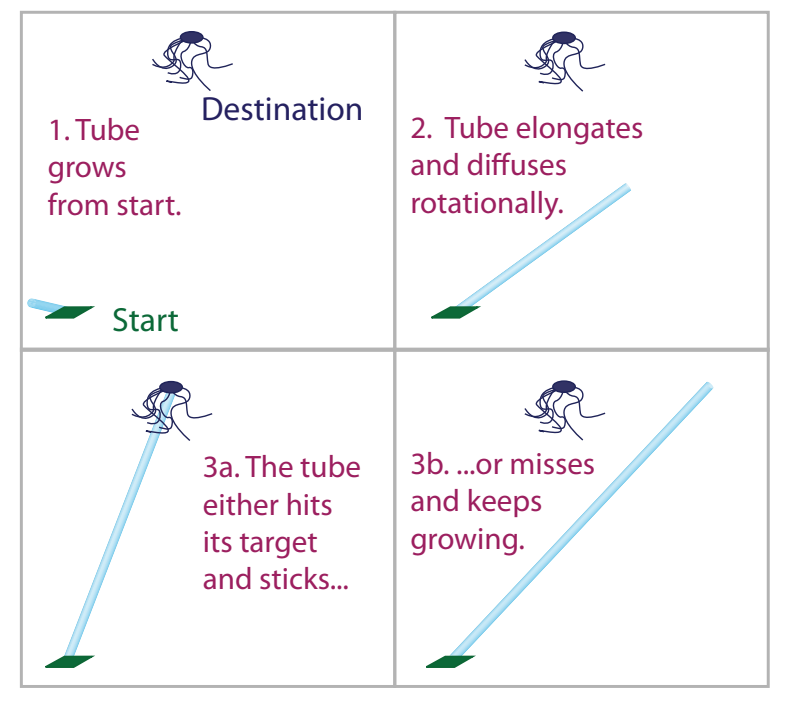

Fig. 1. The path finding algorithm. A stationary start marker attached to a flat surface initiates DNA nanotube growth. A stationary destination marker can bind the end of a growing DNA nanotube. As the DNA nanotube grows (1-2), its end diffuses rotationally, effectively searching for the destination. The DNA nanotube either encounters and sticks to the destination (3a) or gets too long to hit the destination (3b) before finding it.

attached microtubules, separating the chromosomes into two complete sets.

The example of the mitotic spindle suggests that polymer search and capture could be used to form synthetic links between start and destination locations. The mitotic spindle is complex and designed specifically for separating chromosomes, so we propose a simpler molecular algorithm in the same spirit.

The molecules in the path finding algorithm are DNA monomer components that can assemble to form rigid DNA nanotubes (analogous to microtubules), a seed molecule which attaches to the start point (analogous to the centromere), and a capture molecule which attaches to the destination point that growing DNA nanotubes stick to (analogous to the kinetochore). DNA nanotube growth is initiated by a seed [2] at the start point. If the DNA nanotube's end encounters the destination, it sticks and the DNA nanotube forms a path between the start and destination (Figure 1).

Here we seek to understand how this algorithm's performance scales with the size of the destination and the distance between the start and the destination. To do so, we analyze the physics of DNA nanotube diffusion and derive an analytical approximation for the probability that the DNA nanotube will hit the destination. We then determine whether the algorithm will perform well for destination sizes and distances of interest. We use existing estimates for the physical parameters that govern the diffusion and growth rates of DNA nanotubes to numerically compute the probability of forming a path for typical destination sizes and distances from the start point.

In Section II we describe prior work on path finding. In Section III we more formally describe the problem and review the physics of DNA nanotube growth and diffusion that will

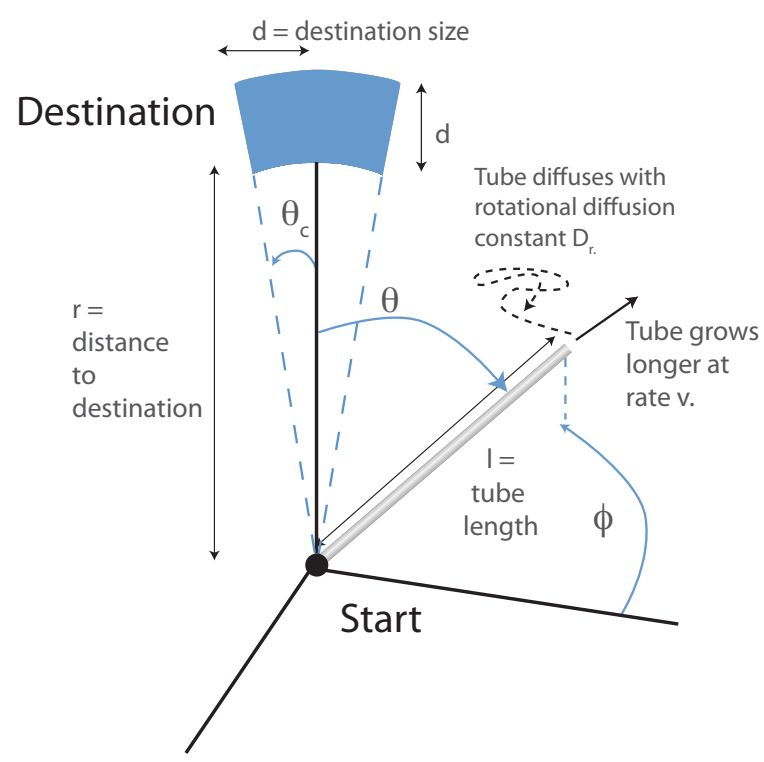

Fig. 2. Schematic for the molecular path finding problem. A start point (black dot) can produce a single growing DNA nanotube (gray cylinder). If the DNA nanotube end enters the destination region $\left(r<l<r+d, \theta<\theta_{c}\right)$, it sticks. The location of the DNA nanotube's end is given using spherical coordinates.

be required to analyze the algorithm's performance. We use this material in Section IV to give expressions for how the algorithm's performance scales with destination distance and size. We show that while path finding via diffusion is very effective for relatively short distances and large destinations, the probability of successful path finding drops exponentially with increasing destination distance and decreasing destination size. In Section V we therefore discuss some possible ways to create algorithms that will perform better when the destination is small and/or far from the start point.

\section{BACKGROUND}

The problem of directing an autonomous agent to find a path through an environment is a classic problem in robotics. Any mobile robot in an uncertain environment must solve some instance of this problem [19]. However, algorithms designed for a single autonomous agent with a large amount of memory and the capacity for efficient, large-scale computation (e.g. [3, 20]) cannot generally be applied to molecular systems.

Path finding has also been studied in biomimetic systems, where large numbers of very simple components with little capacity for computation and memory storage work together to accomplish a goal. In amorphous computing, a collection of fixed, unreliable agents are arrayed randomly in space [1]. Each agent is capable of limited computation and communication with neighboring agents. Paths between specified objects can be discovered in this system using a gradient of morphogens: a destination emits a signal which is passed to neighbors and a path forms in response to these signals [28]. The spatial computing framework, similar to the amorphous computing architecture, provides a language in which path finding can also be solved using gradients [6]. While gradient- 
(a)

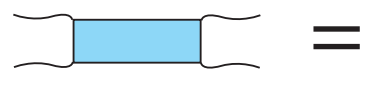

(b)

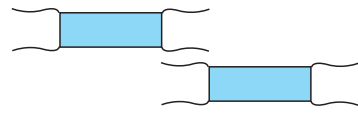

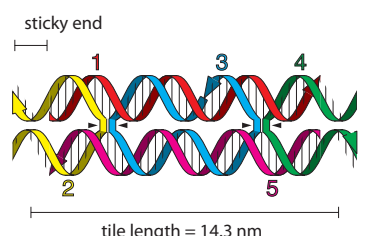

tile length $=14.3 \mathrm{~nm}$

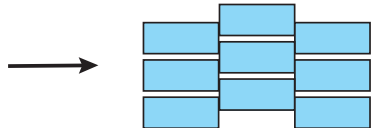

Fig. 3. DAE-E DNA tiles are the building blocks of DAE-E DNA nanotubes. (a) DAE-E DNA tiles consist of 5 short DNA strands that fold into the structure shown on the right because of a preference for WatsonCrick complementarity. (b) Complement sticky end regions hybridize, and the sequences of tiles are designed to hybridize (attach) as shown. Repeated hybridization produces a tile lattice. The particular geometry of DAE-E DNA tiles leads to a slight curved lattice, which is stable as a closed tube [35].

following is efficient and finds paths with high probability, it is still too complex for molecular components to execute.

It is possible to design molecular construction elements with prescribed shape [41,34] and prescribed affinity for other elements $[48,25]$ using synthetic DNA. These construction elements stochastically attach to and detach from each other and move through solution via Brownian motion. DNAbased algorithms for computation and construction exploit the specific affinities of molecules and the fact that when two elements are attached, their affinity for attaching and detaching to certain types other elements can change, which serves as a basic form of state [32, 47, 24].

One widely studied model of molecular construction is the DNA tile self-assembly model $[47,37]$. In this model, DNA components called tiles are equipped with single-stranded sticky ends, and tiles bind to other tiles by these sticky ends, forming lattices (Figure 3). Because tiles can bind only to locations where their sticky ends are complementary to those available, the attachment of a tile to a location where it attaches to two tiles at the same time can be viewed as an information transfer step [47].

The DNA tile assembly model can be used to solve the path finding problem on a 2-dimensional surface, even when obstacles are present [7]. In this method, the start and destination are molecules that interact with tiles. The tiles are designed so that they assemble all possible paths from the start to the destination. The possible paths are attached in a single lattice that eventually integrates the destination molecule. Every tile in the lattice that is part of a complete path is strongly attached to other tiles on the path, while tiles not part of a path are more weakly attached. When the temperature is increased, all attachments are weakened and tiles that are not part of a complete path melt away from the lattice. While this path finding algorithm finds optimal paths using molecular components, the algorithm is not robust to noise in molecular information processing [2] and would be very difficult to implement because it requires a highly structured environment at the molecular scale. The algorithm we describe in this paper
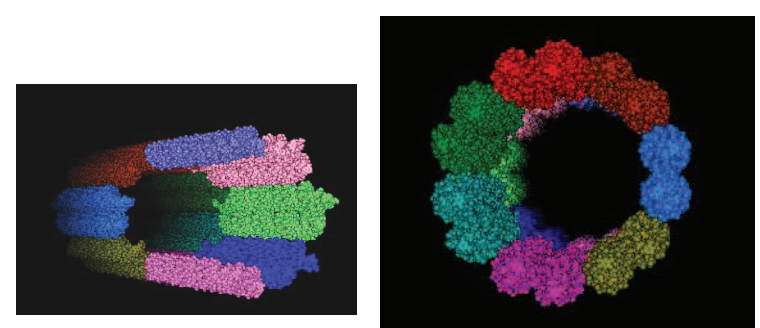

Fig. 4. 3-dimensional models of a 7-tile wide DAE-E DNA nanotube. Each tile is drawn in a different shade. Diagrams were prepared using NAMOT [8] and PyMol [31] software.

is robust, implementable, and also forms 3-dimensional paths in addition to 2-dimensional ones.

\section{PRELIMINARIES}

\section{A. Problem Definition}

Formally, the goal of a path-finding problem is to connect a start location $s$ with a destination region $\mathcal{D}$ by self-assembling a path between them. $s$ is located at the origin and other points are referred to in spherical coordinates, i.e. $(r, \theta, \phi)$.

We will first consider an idealized version of the path finding problem. In the idealized path finding problem, a DNA nanotube grows from $s$ and can diffuse in any angle in three dimensions. The destination object, $\mathcal{D}$ of destination size $d$ is the set of points $\mathcal{D}=\{(p, \theta, \phi)$ such that $r<p<(r+d), \theta<$ $\left.\theta_{c}\right\}$ where $\theta_{c}=\arctan \frac{d}{r+d}$.

In practice, the start and destination objects are attached to large flat surfaces (Figure 1), and DNA nanotubes cannot grow or diffuse through these surfaces. In the implementation version of the path finding problem, a DNA nanotube grows from $s$ and can diffuse only in the region $\theta \leq \pi / 2$.

\section{B. DNA Nanotube Growth}

In this section we describe the construction of DNA nanotubes and how DNA nanotube architecture and the physical conditions for growth determine DNA nanotube growth rates.

We consider the growth of DAE-E DNA tile nanotubes. DAE-E DNA tile nanotubes consist of DAE-E DNA tiles (Figure 3) that assemble into a structure consisting of a radial array of parallel DNA helices (Figure 4). A DAE-E DNA tile consists of 5 short, synthetic DNA strands and is 14.3 nanometers long and about 4 nanometers wide. DAEE DNA tiles attach via the hybridization of the single strand sticky ends on their four edges. A DAE-E DNA tile nanotube with $n$ helices has $n / 2$ tiles in each layer and in practice, DAE-E DNA nanotubes with 8-24 helices are known to be stable [35]. In this paper, DAE-E DNA tiles and DAE-E DNA tile nanotubes will be henceforth referred to simply as DNA tiles and DNA nanotubes.

Experiments with DNA tiles [36, 38, 29] support a model where tile attachment and detachment rates are first order chemical reactions [48]. The rate $k_{o n}$ at which a tile attaches at such a site is given by

$$
k_{o n}=k_{f}[t]
$$


where $k_{f}$ is a diffusion controlled forward rate [45] and [t] is the concentration of the tiles in the environment. This rate means that tiles arrive at an attachment site at exponentially distributed times, where the mean is $k_{o n}$. A tile attached to a nanotube by $n$ sticky end bonds detaches from the DNA nanotube at rate $k_{o f f, n}$ :

$$
k_{o f f, n}=k_{f} e^{-\frac{\Delta G_{n}}{R T}}
$$

where $\Delta G_{n}$ is the Gibbs free energy of attachment by $n$ sticky end bonds, $R$ is the universal gas constant, and $T$ is absolute temperature. Sticky end binding is cooperative, so that $\Delta G_{n}$ increases with $n[33,9,29]$.

When $k_{o f f, 2}<k_{o n} \ll k_{o f f, 1}$, i.e. when the attachment of a tile to a DNA nanotube is favorable only if the tile attaches by at least 2 bonds, DNA nanotubes grow quickly from a seed structure where tiles can attach by 2 bonds, but slowly in free solution, where attachments by just 1 bond are required to initiate the growth of a new DNA nanotube [14, 38, 39]. Under these conditions, DNA nanotubes grow by the attachment of tiles at the DNA nanotube's distal end, and most attaching tiles form two sticky end bonds.

We used a simple stochastic kinetic simulation of DNA nanotube growth $[15,48]$ to estimate nanotube growth rates. In the simulation, a tile could attach to a DNA nanotube in a location where it formed two bonds and detach from a DNA nanotube if it was attached by no more than two bonds. We used experimentally measured values of $k_{f}, \Delta H$ and $\Delta S$ in the simulation. $k_{f}$ for a similar type of DNA tiles has been measured and is approximately 3,000,000 per Molar per second, where Molar is moles per liter [17]. $\Delta G=$ $\Delta H-T \Delta S$ has been measured for similar DNA tiles, where $T$ is absolute temperature. $\Delta H=-102.4$ kilocalories $/ \mathrm{mol}$ and $\Delta S=-0.300$ kilocalories / mol [38]. Our estimate for the growth rate of a 12-tile-wide DNA nanotube in tile rows per unit time was the average time it took to grow a row in the simulated growth of about 2000 tiles. For experiments that take place at around 305 Kelvin and $40 \mathrm{nM}$ tile concentration, we found that a 6-tile wide DNA nanotube grows $14.3 \mathrm{~nm}$ (the length of 1 tile row) about every $94.2 \pm 1.6$ seconds (1.6 seconds is 2 standard deviations of the predicted time per row in 100 iterations of this simulation). We used this value for the growth rate $v$ in the next section. The fluctuations in length due to the stochastic nature of growth should be on order the square root of the DNA nanotube length (in tile rows) and were ignored in our analysis. The growth rate can be made faster or slower by increasing or decreasing the tile concentration.

\section{Polymer Dynamics}

Previous studies indicate that DAE-E DNA nanotube stiffness and thus diffusion rates are consistent with existing polymer physics models. This section describes the basic components of these models that are used in the analysis of the path finding algorithm in the next section.

Because a polymer's repeating units are physically connected, the directions of the units' Brownian motion are correlated, and the degree of correlation in this motion is the polymer's stiffness. The basic unit of polymer stiffness is its persistence length, formally the length scale over which correlations in the direction of the polymer's tangent vector are lost. The persistence length of a polymer is intrinsic to its repeating structure, i.e. there is a persistence length for double-stranded DNA, which is $p_{\text {helix }}=50$ nanometers [42]. Assuming that the Young's modulus of a DNA helix and a DNA nanotube are the same, the persistence length of a DNA nanotubes is predicted to be [35]:

$$
\frac{p_{\text {tube }}}{p_{\text {helix }}}=2 N\left[1+2\left(\frac{R}{r}\right)^{2}\right]
$$

where $p_{\text {tube }}$ is the persistence length of the nanotube, $N$ is the number of helices in the nanotube, $R$ is the nanotube radius and $r \approx 1$ nanometer is the radius of a DNA double helix. This equation predicts that 6-tile-wide to 12-tile-wide nanotubes should have a persistence lengths from 10 to 80 $\mu \mathrm{m}$ respectively. These values are qualitatively consistent with experimentally measured values of nanotube persistence length [35, 14]. The width of the DNA nanotubes used in the path finding algorithm can be controlled by designing the seed for the DNA nanotube's to grow DNA nanotubes of the desired width [2]. It seems feasible to make DNA nanotubes with a persistence length of up to 12 tiles around, or $\approx 80$ $\mu \mathrm{m}$.

When a polymer is much shorter than its persistence length, it is classified as rigid and behaves much like a straight rod. Since in our path finding algorithm one end of the growing nanotube is attached to the start point, a rigid nanotube diffuses by rotation only. In this case, if a DNA nanotube has length $l$, the end of the DNA nanotube is effectively diffusing around the surface of a sphere with radius $l$. The diffusion speed is characterized by a rotational diffusion constant, which we will call $D_{\text {rot }}$. If a nanotube has length $l$ and the location of a nanotube's end point at time $t$ is $u(t)$, then $\left\langle(u(t)-u(0))^{2}\right\rangle=$ $4 D_{\text {rot }} l^{2} t$ [12]. Here we consider tube lengths up to $25 \mu \mathrm{m}$, which is much less than the $80 \mu \mathrm{m}$ persistence length of some DNA nanotubes, so we assume that the diffusing tube is rigid.

The rotational diffusion constant of an isolated polymer in a fluid appears to be dependent mostly on the polymer's size rather than its molecular type [50], and enough information about rotational diffusion constant exists to allow us to predict the changes that DNA nanotubes will find their destination in this regime.

\section{PATH Finding EFFiCIENCY}

\section{A. Analysis of Path Finding Efficiency in an Idealized Geom- etry}

We are interested in the question of whether a DNA nanotube finds its destination via diffusion as it grows. When a DNA nanotube length's $l$ is less than the length to the edge of the destination $r$, the probability of the DNA nanotube's end hitting the destination is 0 (Figure 2). Similarly, when the DNA nanotube's end is longer than $r+d$, where $d$ is the destination size, the probability of the DNA nanotube's end 
hitting the destination is also 0 . We are therefore interested in the chance that the DNA nanotube finds the destination when $r \leq l \leq r+d$. The time during which the DNA nanotube's length is in this regime is $t=d / v$, where $v$ is the growth speed of the DNA nanotube.

If $d_{\text {tube }}$ is the DNA nanotube's cross-sectional diameter, $T$ is absolute temperature, $k_{b}$ is Boltzmann's constant and $\eta_{s}$ the is viscosity of the solution the DNA nanotube is grown in, the rotational diffusion constant (in distance squared per time) is approximately [50]:

$$
\begin{array}{rc}
D_{\text {rot }} & =\frac{3 k_{b} T}{\pi \eta_{s} l}(\delta-\zeta) \\
\delta & =\log \left(\frac{2 l}{d_{\text {tube }}}\right) \\
\zeta=1.45-7.5\left(\frac{1}{\delta}-0.27\right)^{2}
\end{array}
$$

where $\delta$ and $\zeta$ are hydrodynamic correction factors. When $l<r$, the probability density that the DNA nanotube is oriented at angles $(\theta, \phi)$ is uniform, i.e. $\rho_{l}(\theta, \phi)=\frac{1}{4 \pi l^{2}}$. If $\theta<\theta_{c}=\arctan \frac{d}{r+d}$ when the DNA nanotube first grows to a length $l \geq r$, the DNA nanotube immediately attaches to the destination and has succeeded in building a path. The probability that the DNA nanotube hits immediately, $P_{0}$ is

$$
\begin{aligned}
P_{0}\left(r, \theta_{c}\right) & =\int_{0}^{2 \pi} \int_{0}^{\theta_{c}} r^{2} \sin (\theta) \rho_{r}(\theta, \phi) \\
& =\frac{1}{2}\left(1-\cos \left(\theta_{c}\right)\right)
\end{aligned}
$$

If upon reaching length $r$ the DNA nanotube does not hit the destination immediately, the probability density of its orientation is uniform over all points $\theta>\theta_{c}$. We can use the diffusion equation over the probability distribution that a DNA nanotube is located at some angle (i.e. the Smoluchowski equation) to determine the fraction of DNA nanotubes that find the destination by diffusing into it, $P_{d i f f}$.

The diffusion equation in spherical coordinates is given by

$\frac{\partial \rho}{\partial t}=\frac{D}{r^{2}}\left[\frac{\partial}{\partial r}\left(r^{2} \frac{\partial \rho}{\partial r}\right)+\frac{1}{\sin ^{2} \theta} \frac{\partial^{2} \rho}{\partial \phi^{2}}+\frac{1}{\sin \theta} \frac{\partial}{\partial \theta}\left(\sin \theta \frac{\partial \rho}{\partial \theta}\right)\right]$

Because we ignore the small fluctuations in DNA nanotube length, there is no diffusion in the $\hat{\mathbf{r}}$ direction, and because the destination is radially symmetric with respect to $\hat{\phi}, \rho$ is independent of $\phi$. The diffusion equation therefore simplifies to:

$$
\frac{\partial \rho}{\partial t}=\frac{D_{r o t}(r)}{r^{2} \sin (\theta)} \frac{\partial}{\partial \theta}\left(\sin (\theta) \frac{\partial \rho}{\partial \theta}\right)
$$

with boundary conditions

$$
\begin{gathered}
\rho=0 \text { at } \theta=\theta_{c} \\
\frac{\partial \rho}{\partial \theta}=0 \text { at } \theta=\pi
\end{gathered}
$$

The boundary conditions stipulate that if a DNA nanotube hits the destination it is absorbed, and if it hits the pole opposite the destination it continues to diffuse.

In the case where $r \gg d$, i.e. where the destination is small and far away, the diffusion constant does not change significantly over the time that the DNA nanotube could hit it. In this case, $D_{\text {rot }}$ is approximately constant for $r \leq l \leq$ $r+d$, and the time it takes before the DNA nanotube first diffuses into the destination is approximately exponentially distributed [44] with mean [22]:

$$
\left\langle t_{\text {hit }}\right\rangle \approx 2 \frac{r^{2}}{D_{\text {rot }}}\left[\frac{\log \frac{2}{1-\cos \theta_{c}}}{1+\cos \theta_{c}}-1\right]
$$

The length of time during which a DNA nanotube can hit the destination is $t_{\max }=d / v$. The probability that the DNA nanotube diffuses into the destination, $P_{\text {diff }}$, is therefore

$$
P_{\text {diff }} \approx 1-\exp \left[\frac{t_{\max }}{\left\langle t_{\text {hit }}\right\rangle}\right],
$$

so the total probability that the DNA nanotube successfully finds the destination is

$$
P_{\text {hit }} \approx P_{0}+\left(1-P_{0}\right) P_{\text {diff }} .
$$

Combining Equations 14 and 13 gives

$$
P_{\text {diff }} \approx 1-\exp \left[\frac{-d / v}{2 \frac{r^{2}}{\frac{3 k_{b} T}{\pi \eta_{s} l}(\delta-\zeta)}\left[\frac{\log \frac{2}{1-\cos \theta_{c}}}{1+\cos \theta_{c}}-1\right]}\right]
$$

Some algebra simplifies this equation to

$$
P_{d i f f} \approx 1-\exp \left[\frac{-d C(\delta-\zeta)}{v r^{2} l\left[\frac{\log \frac{2}{1-\cos \theta_{c}}}{1+\cos \theta_{c}}-1\right]}\right]
$$

where $C=\frac{3 k_{b} T}{2 \pi \eta_{s}}$, a constant that does change with either destination distance or size.

Figures 5 and 6 show $P_{\text {hit }}$ for the rotational diffusion constant in Equation 4 and the DNA nanotube growth rate given in Section III. The viscosity of the solution was assumed to be the viscosity of water at $305 \mathrm{~K}, 7.98 \times 10^{-4}$ $\mathrm{Pa} \cdot \mathrm{s}$. Probabilities were computed via numerical integration of Equation 10 and its approximate analytical solution given in Equation 17. The results of numerical integration and of the analytical approximation are similar and both methods suggest that for short distances $(<10 \mu \mathrm{m})$, virtually all DNA nanotubes should find a destination with a diameter of 500 nanometers. The probability of finding the destination drops sharply as both $r$ increases and $d$ decreases, however.

To quantitatively determine how the probability of finding the destination scales with increasing $r$ and decreasing $d$, note that $\left(\frac{\log \frac{2}{1+\cos \theta_{c}}}{1+\cos \theta_{c}}-1\right)$ increases as $\theta_{c}$ decreases. $P_{\text {diff }}$ therefore decreases with decreasing $\theta_{c}$. In contrast, the factor $\delta-\zeta$ increases with $\log r$.

Thus, as $r$ increases for constant $d, 1-P_{\text {diff }}$ increases as $\exp \left[-1 / \Omega\left(r^{3}\right)\right]$. That is, as the destination becomes further away, the probability of a DNA nanotube hitting the destination before growing too long to do so drops exponentially as the cube of the distance! As $d$ decreases for a constant destination distance $r, 1-P_{\text {diff }}$ scales as approximately $\exp [-\Omega(d)]$. Since $P_{0} \ll P_{\text {diff }}$ for all but the closest, largest destinations, $1-P_{h i t}$ scales approximately as $1-P_{d i f f}$. 


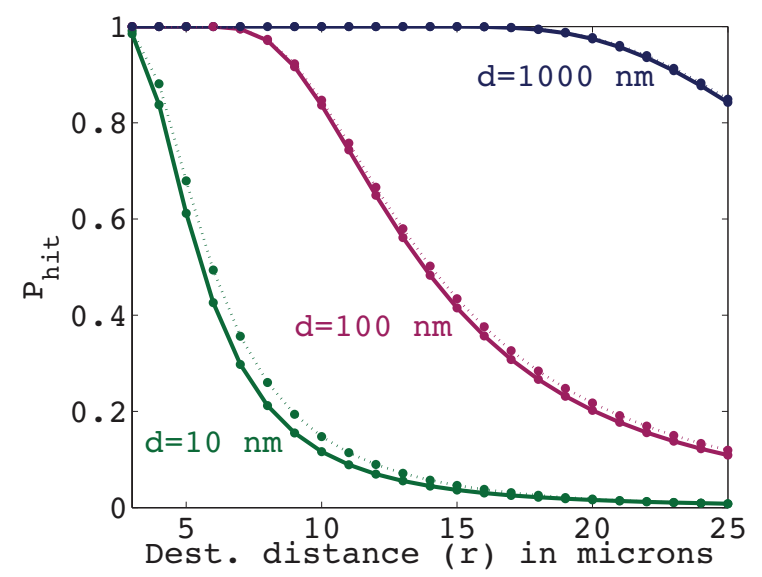

Fig. 5. Predicted performance of the path finding algorithm as the destination distance $r$ varies for destination sizes $d=10 \mathbf{n m}, d=100 \mathbf{n m}$ and $d=1000 \mathrm{~nm}$. Solid lines show the approximate analytical solution to Equation 17. Dashed lines are the numerical integration of Equation 10.

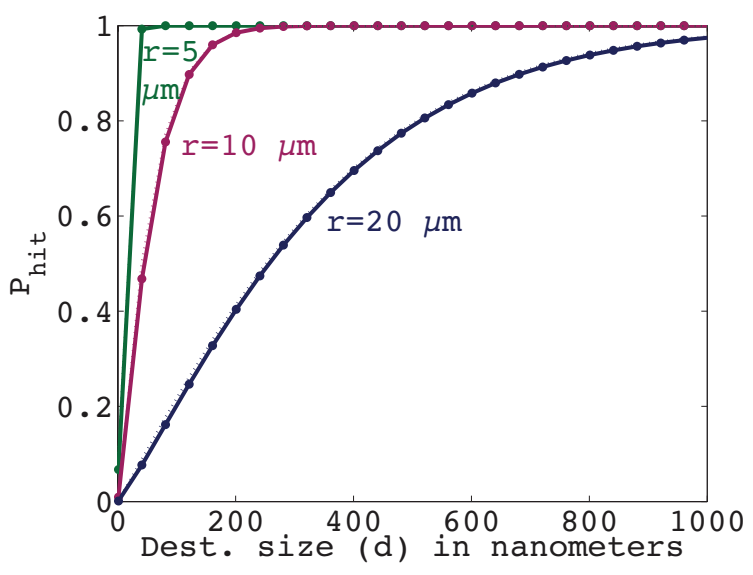

Fig. 6. Predicted performance of the path finding algorithm as the destination size $d$ varies, for destination distances $r=5 \mu \mathrm{m}, r=10 \mu \mathbf{m}$ and $r=20 \mu \mathrm{m}$. Solid lines show the approximate analytical solution to Equation 17. Dashed lines are the numerical integration of Equation 10, but are too close to the analytical solution to be distinguished.

While the chances of hitting the destination decrease exponentially with increasing destination distance or decreasing destination size, decreasing the speed of DNA nanotube growth can drastically improve the performance. $\log \left(1-P_{\text {diff }}\right)$ changes as $\Theta(1 / v)$. That is, halving the speed of growth roughly squares the probability of missing the destination.

\section{B. Simulation of Path Finding in an Implementation Geometry}

In a path finding implementation, the start and destination markers are attached to solid objects that prevent a growing DNA nanotube from moving freely in every direction. Additionally, the geometry of the destination marker is unlikely to have a shape, as shown in Figure 2, where for each length $l$ in the range $r<l<r+d$, the extent of the destination structure is described by the same $\theta_{c}$. These features of

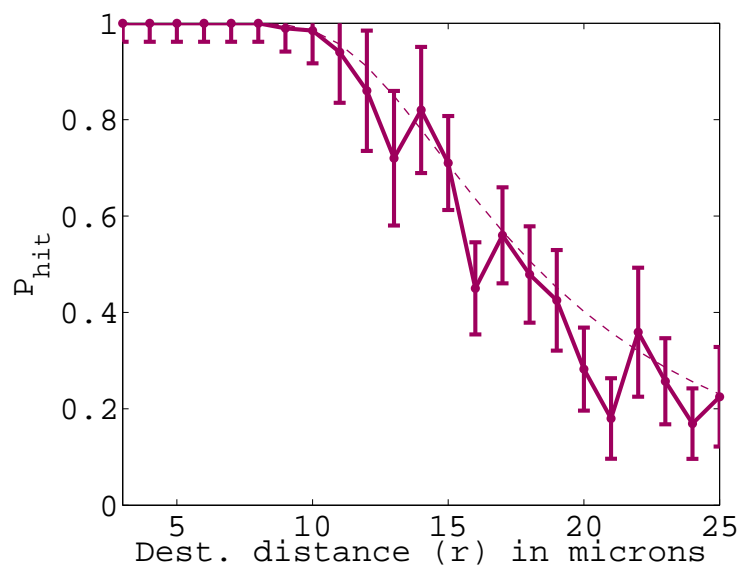

Fig. 7. Simulated probability of hitting a target in the implementation geometry. The thick line shows the simulated probability of hitting a destination, a half sphere of radius $100 \mathrm{~nm}$ located on the same flat surface as the start point. Error bars are two standard deviations. The dashed line is the approximate analytical values of $P_{h i t}$ for the idealized geometry with destination size $200 \mathrm{~nm}$.

an implementation can change the rate and pattern of DNA nanotube end diffusion, and therefore path finding efficiency.

We developed a stochastic simulation of DNA nanotube diffusion to predict path finding efficiency in geometries containing irregular environment and destination marker geometries. We tested this simulation on a particularly simple implementation geometry where both the start and markers are attached to a solid surface and where the DNA nanotube is otherwise free to diffuse.

The destination in the implementation geometry is a half sphere with radius $100 \mathrm{~nm}$ centered on the surface. The seed size is $100 \mathrm{~nm}$ and DNA nanotubes were 12 tiles wide. DNA nanotubes can diffuse in any direction that did not collide with the surface to which the destination and surface markers were attached. The temperature, tile concentration, and sample viscosity in the simulation were the same as those used in Section IV-A.

The stochastic kinetic attachment and detachment of tiles governed DNA nanotube length as described in Section IIIB. Diffusion was modelled via a standard algorithm for discretized Brownian dynamics on a sphere [12] where diffusive motion vectors were generated by moving the DNA nanotube a randomly angle on the plane tangent to the sphere and projecting the final point back onto the surface of the sphere. We used a time step of $10 \mu \mathrm{s}$, or an average DNA nanotube movement of 11-12 nm per time step for $l=3$ microns. Approximately 100 DNA nanotubes were simulated per destination distance.

The results of the simulations are given in Figure 7. $P_{\text {hit }}$ in the implementation geometry scales similarly to $P_{h i t}$ in the idealized geometry, but the absolute probability is slightly less in the simulated implementation geometry than in the idealized geometry. Both of these results are to be expected. Unless an obstacle prevents the DNA nanotube from reaching the destination (in which case the probability of forming a path 
is 0 ), the efficiency is related to the ratio of the volume that the DNA nanotube's end can diffuse through and the volume covered by the destination. This ratio scales at the same rate for both the idealized geometry and the implementation geometry. In the implementation geometry, the probability of hitting the destination, a half sphere, changes significantly for $r-d \leq$ $l \leq r+d$, whereas in the idealized geometry, the probability of the tube hitting the target does not change over this length interval. Because the overall volume of the destination in the implementation geometry is smaller than the volume of the destination in the idealized the geometry, the probability of hitting is slightly less in the implementation geometry.

\section{Conclusions ANd Future Directions}

We've described an algorithm for assembling a molecular path between two points and used analysis and simulations to determine how the algorithm's probability of success scales with the size of the destination and its distance from the start point. The probability that the self-assembly process will complete when the necessary path is short (less than 10 microns) and the destination is large (more than 500 nanometers in radius) are high, meaning that the algorithm is practical for many situations.

However, we've also shown that the performance of the algorithm falls off superexponentially as the distance to the destination increases and at least exponentially as the destination size decreases. These performance drop-offs are not optimal: a linear search for non-growing tubes would complete successfully with probability 1 in a time that decreased quadratically as the destination distance increased and as the destination size decreased. It is therefore worth considering mechanisms that would improve the performance of the search process. It may be difficult to do so with the existing capacities of designed molecules, but an evaluation of the kinds of information processing that would provide a qualitative improvement in path finding performance could be used to guide the development of new molecular components.

The current algorithm's exponential performance decreases are caused by the random search for the destination and the limited time window during which a DNA nanotube can find and attach to a destination. Speeding up or changing the diffusion process would therefore simply change the exponent of performance falloff by a constant factor. But if a DNA nanotube could specifically grow toward the destination, performance might be improved. This would be possible if the destination could somehow communicate information to the growing DNA nanotube about its location in advance of contacting it. This type of communication occurs during growth of the Xenopus laevis mitotic spindle, where a gradient of small molecules forms around the destination for microtubule attachment [4]. One might be tempted to use such a gradient to increase the DNA nanotube's growth rate as it neared the destination, but such a mechanism might actually decrease the probability of finding the destination, because as the DNA nanotube approached the destination it would grow faster and

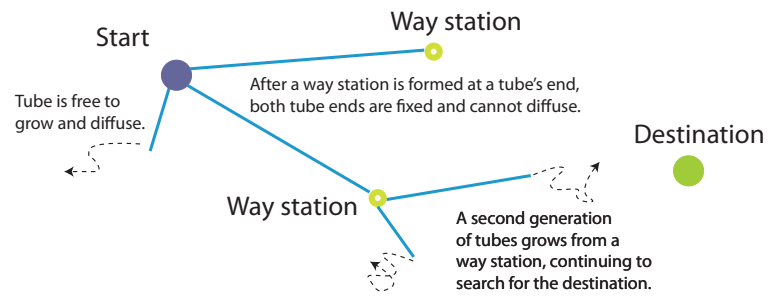

Fig. 8. A divide and conquer approach to molecular path finding. Instead of a single DNA nanotube growing and looking for the destination by diffusion, an initial set of DNA nanotubes could each stop growing and create fixed "way stations" from which a second set of DNA nanotubes could grow. The arrangement of way stations would be distributed evenly in space if each way station emitted a gradient preventing other way stations from forming nearby. This process could happen recursively, allowing DNA nanotubes to locate faraway destinations efficiently.

faster, decreasing the time available for searching for the destination.

In Xenopus laevis, the gradient directs microtubule growth via a more subtle mechanism. Microtubules exhibit dynamic instability -i.e. they grow with a constant rate for a period of time, and then occasionally undergo catastrophes, periods of abrupt shrinking [27]. By allowing length decreases as well as increases, dynamic instability would improve the chances that a DNA nanotube would find the destination eventually, by giving the DNA nanotube more than one chance to explore the area that is the right distance from the start point. Dynamically unstable growth combined with a gradient of molecules emanating from the destination that decreased the frequency of catastrophe might create a qualitative improvement in the probability of finding the destination: a DNA nanotube would remain short, diffusing quickly, until it found the generally correct direction to hunt for the destination where the concentration of molecules that reduced the probability of catastrophes was highest [49]. So long as the DNA nanotube continued to diffuse in an area where the concentration of the catastrophe-reducing molecule was high, it would continue to grow. Further, if the DNA nanotube missed the destination, it would undergo catastrophes that would shorten the DNA nanotube until it became approximately a length that could reach the destination.

Another possibility would be to consider parallel searching. In one such approach, the DNA nanotubes could occasionally attach to a nearby surface and stop growing, creating a "way station" from which several new DNA nanotubes could begin growing. If way stations emitted gradient signals discouraging the creation of other way stations nearby, DNA nanotubes might distribute such stations evenly in space (Figure 8). A way station would have the effect of speeding up the search because DNA nanotubes growing from the way stations would be shorter than DNA nanotubes that had grown all the way from the start point and therefore would diffuse more quickly. However, this algorithm's application would be limited to environments where surfaces or other stationary structures were available for way stations to attach to. Implementing a clean-up process to remove way stations and DNA nanotubes 
that are not part of a successful path would also be necessary.

\section{REFERENCES}

[1] Abelson, H., Allen, D., Coore, D., Hanson, C., Homsy, G., Knight, T. F. Nagpal, R., Rauch, E., Sussman, G. J., Weiss, R. "Amorphous Computing," Communiciations of the ACM, vol. 43, iss. 5, pp 74-82, 2000.

[2] Barish, R., Schulman, R., Rothemund, P. W. K., Winfree E. "An Information-Bearing Seed for Nucleating Algorithmic Self-Assembly," Proceedings of the National Academy of Sciences USA, vol. 106, no. 15, pp 6054-6059, 2009.

[3] Barraquand, J., Langlois, B., Latombe, J. "Numerical Potential Field Techniques for Robot Path Planning," IEEE Transactions on Systems, Man and Cybernetics, vol. 22, no. 2, pp 224-241, 1992.

[4] Bastiaens, P., Caudron, M., Niethammer, P., Karsenti, E. "Gradients in the self-organization of the mitotic spindle," Trends in Cell Biology, vol. 16, no. 3, pp. 125-134, 2006.

[5] Bath, J., Green, S. J., Turberfield, A. J. "A free-running DNA motor powered by a nicking enzyme," Angewandte Chemie International Edition, vol. 44, pp. 4358-4361, 2005.

[6] Beal, J., Bachrach, J., "Infrastructure for engineered emergence on sensor/actuator networks," IEEE Intelligent Systems, vol 21, iss. 2, pp 10-19, Mar. 2006.

[7] Brun, Y. and Reichus, D. "Path finding in the tile assembly model," Theoretical Computer Science, vol. 410, pp 1461-1472, 2009.

[8] Carter, II, E. S., Tung, C.-S. "NAMOT2 - a redesigned nucleic acide modelling tool: construction of non-canonical DNA structures," Bioinformatics, vol. 12, no. 1, pp 25-30, 1996.

[9] Chen, H.-L., Schulman, R., Goel, A., Winfree, E. "Preventing Facet Nucleation During Algorithmic Self-Assembly," Nano Letters, vol. 7, no. 9, pp. 2912-2919, 2007.

[10] Chen, H.-L., Goel, A. "Error Free Self-Assembly using Error Prone Tiles," DNA Computing 10, Milan, It., pp 62-75, Jun. 2004.

[11] Cook, M., Soloveichik, D., Winfree, E., Bruck, J. "Programmability of Chemical Reaction Networks," Algorithmic Bioprocesses, Berlin Heidelberg: Springer, 2009, pp 543-584.

[12] Doi, M. and Edwards, S. F., The Theory of Polymer Dynamics. Oxford, England: Oxford University Press, 1986.

[13] Douglas, S. M., Dietz, H., Liedl, T., Högberg, B., Graf, F., Shih, W. M. "Self-assembly of DNA into nanoscale three-dimensional shapes," Nature, vol. 459, pp 414-418, 2009.

[14] Ekani-Nkodo, A., Kumar, A., Fygenson, D. K. "Joining and Scission in the Self-Assembly of Nanotubes from DNA Tiles," Physical Review Letters, vol. 93, pp 268301, 2004.

[15] Gillepsie, D. T., "Exact stochastic simulation of coupled chemical reactions," Journal of Physical Chemistry, vol. 81, no. 25, pp. 23402361, 1977.

[16] Gu, H., Chao, J., Xiao, S.-J., Seeman, N. C. "A proximity-based programmable DNA nanoscale assembly line," Nature, vol. 465, pp. 202205, 2010.

[17] Hariadi, R., Yurke, B., Winfree E. Mauscript in preparation.

[18] Karsenti, E.,Vernos, I. "The Mitotic Spindle: A Self-Made Machine," Science, vol. 294, pp 543-547, 2001.

[19] Latombe, J. Robot Motion Planning, Dordrecht, Neth.: Kluwer Academic, 1990

[20] LaValle, S. M. Planning Algorithms, Cambridge, UK: Cambridge University Press, 2006.

[21] Lehn, J.-M., "Supramolecular Chemistry," Science, vol. 260, iss. 5115, pp. 1762-1764, 1993.

[22] Linderman, J. J., Lauffenberger, D. A. "Analysis of Intracellular Receptor/Ligand Sorting: Calculation of Mean Surface and Bulk Diffusion Times within a Sphere," Biophysical Journal, vol. 50, pp 295-305, 1986.

[23] Lund, K., Manzo, A. J., Dabby, N., Michelotti, N., Johnson-Buck, A., Nangreave, J., Taylor, S., Pei, R., Stojanovic, M., Walter, N. G., Winfree, E., Yan, H. "Molecular robots guided by prescriptive landscapes," Nature, vol. 465, pp. 206-210, 2010.

[24] Magnasco, M. O. "Chemical Kinetics is Turing Universal," Physical Review Letters, vol. 78, no. 6, pp. 1190-1193, 1997.
[25] Mao. C., LaBean, T. H., Reif, J. H., Seeman, N. C. "Logical Computation using algorithmic self-assembly of DNA triple-crossover molecules," Nature, vol. 407, no. 6803, pp. 493-496, 2000.

[26] Markov, I. V. Crystal Growth for Beginners, Singapore: World Scientific, 2003.

[27] Mitchison, T., Kirschner, M. "Dynamic instabiity of microtubule growth," Nature, vol. 312, pp. 237-242, 1984.

[28] Nagpal, R. "Programmable Self-Assembly Using Biologically-Inspired Multiagent Control." Conference on Autonomous Agents and Multiagent Systems (AAMAS), Bologna, It., July 2002.

[29] Nangreave, J., Yan, H., Liu, Y. "Studies of Thermal Stability of Multivalent DNA Hybridization ina Nanostructured System," Biophysical Journal, vol. 97, iss. 2, pp 563-571, 2009.

[30] Omabegho, T., Sha, R., Seeman, N. C. "A Bipedal DNA Brownian motor with coordinated legs," Science vol. 324, pp. 67-71, 2009.

[31] The PyMOL Molecular Graphics System, Version 1.3, Schrdinger, LLC.

[32] Rothemund, P. W. K. "A DNA and Restriction Enzyme Implementation of Turing Machines," DNA Based Computers, Princeton, NJ, pp. 75-119, Apr. 1995.

[33] Rothemund, P. W. K. "Theory and Experiments in Algorithmic SelfAssembly," PhD thesis. University of Southern California, Los Angeles, 2001.

[34] Rothemund, P. W. K. "Folding DNA to create nanoscale shapes and patterns," Nature, vol. 440, pp. 297-302, 2006.

[35] Rothemund, P. W. K., Ekani-Nkodo, A., Papadakis, N., Kumar, A., Fygenson, D. K., Winfree, E. "Design and Characterization of Programmable DNA Nanotubes," Journal of the Americal Chemical Society, vol. 126, no. 50, pp 16344-16353, 2004.

[36] Rothemund, P. W. K., Papadakis, N., Winfree, E. "Algorithmic SelfAssembly of DNA Sierpinski Triangles," PLoS Biology, vol. 2, iss. 12, pp 424-436, 2004.

[37] Rothemund, P. W. K., Winfree, E. "The Program-Size Complexity of Self-Assembled Squares," Symposium on Theory of Computing (STOC), Portland, Or., pp 459-468, May 2000.

[38] Schulman, R., Winfree, E. "Synthesis of crystals with a programmable kinetic barrier to nucleation," Proceedings of the National Academy of Sciences USA, vol. 104, no. 39, pp 15236-15241, 2007.

[39] Schulman, R., Winfree, E. "Programmable Control of Nucleation for Algorithmic Self-Assembly," SIAM Journal on Computing, vol. 39, iss. 4, pp 1581-1616, 2009.

[40] Seelig, G., Soloveichik, D., Zhang, D. Y., Winfree, E. "Enzyme-Free Nucleic Acid Logic Circuits," Science, vol. 314, pp. 1585-1588, 2006.

[41] Seeman, N. C. "An Overview of Structural DNA Nanotechnology," Molecular Biotechnology, vol. 37, no. 3, pp 246-257, 2007.

[42] Smith, S. B., Finzi, L., Bustamante, C. "Direct mechanical measurements of the elasticity of single DNA molecules by using magnetic beads," Science, vol. 258, pp. 11221126, 1992.

[43] Soloveichik, D., Winfree, E., "Complexity of self-assembled shapes," SIAM Journal on Computing, vol. 36, no. 6, pp 1544-1569, 2007.

[44] Szabo A., Schulten, K., Schulten, Z. "First passage time approach to diffusion-controlled reactions," Journal of Chemical Physics, vol. 72, pp. 4350-4357, 1980.

[45] Wetmur, J. G., Fresco, J. "DNA Probes: Applications of the Principles of Nucleic Acid Hybridization," Critical Reviews in Biochemistry and Molecular Biology, vol. 26, no. 3-4, pp 227-259, 1991.

[46] Whitesides, G. M., Mathias, J. P., Seto, C. "Molecular self-assembly and nanochemistry: a chemical strategy for the synthesis of nanostructures," Science, vol. 254, iss. 5036, pp 1312-1319, 1991.

[47] Winfree, E. "On the Computational Power of DNA Annealing and Ligation," DNA Based Computers, Princeton, NJ, pp. 187-198, Apr. 1995.

[48] Winfree, E. "Simulations of Computing by Self-Assembly," California Institute of Technology Technical Report CS-TR:1998.22, 1998.

[49] Wollman, R., Cytrynbaum, E. N. Jones, J. T., Meyer, T. Scholey, J. M., Mogilner, A., "Efficient chromosome capture requires a bias in the "Search-and-Capture" process during mitotic spindle assembly", Current Biology, vol. 15, pp. 828-832, 2005.

[50] Zero, K. M., Pecora, R. "Rotational and Translational Diffusion in Semidilute Solutions of Rigid-Rod Macromolecules," Macromolecules, vol. 15, pp 87-93, 1982. 